\title{
Formulation of Kaempferol in Nanostructured Lipid Carriers (NLCs): A Delivery Platform to Sensitization of MDA-MB468 Breast Cancer Cells to Paclitaxel
}

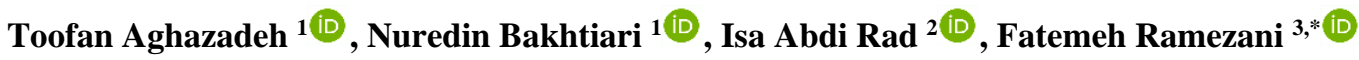 \\ 1 Department of Biochemistry, Faculty of Life Sciences, Tehran North Branch, Islamic Azad University, Tehran, Iran; \\ Toofanaghazade@gmail.com (T.A.); Nuredin1480@gmail.com (N.B.); \\ 2 Cellular and Molecular Research Center, Cellular and Molecular medicine Institute, Urmia University of Medical \\ Sciences, Urmia, Iran; Isaabdirad@yahoo.com (I.A.R.); \\ 3 Department of Molecular Medicine, School of Advanced Medical Science, Tabriz University of Medical Sciences, Tabriz, \\ Iran; Framezani82@gmail.com (F.R.); \\ * Correspondence: Framezani82@gmail.com;
}

Scopus Author ID: 57193232842

Received: 6.02.2021; Revised: 4.03.2021; Accepted: 7.03.2021; Published: 25.03.2021

\begin{abstract}
Drug delivery-based nanoparticle has been developing as a widespread innovation in cancer treatment protocols. Here, we investigated the role of Kaempferol (KAE) loaded in nanostructured lipid carriers (NLCs) to promote cytotoxicity, efficacy, and paclitaxel-dependent apoptosis in MDA-MB 468 breast cancer cells. Particle size distribution, scanning electron microscopy (SEM), zeta potential, and cellular uptake were harnessed to optimize and characterize of KAE -loaded NLCs. MTT assay was used to measure the cellular proliferation of cancer cells. The clarification of early and late apoptosis and their gene expression patterns was assessed by Annexin V/PI staining and real-time PCR, respectively. SEM images offered us a nasty particle size of $80 \pm 3 \mathrm{~nm}$ to the Kaempferol formulated into NLCs. The $\mathrm{IC}_{50}$ values for KAE and paclitaxel determined $44 \pm 0.52 \mu \mathrm{M}$ and $1.75 \pm 0.36 \mathrm{nM}$, respectively. The moderated cell proliferation from $56 \pm 26.8 \%$ to $44 \pm 3.9 \%$ (p< $<0.05$ ) was demonstrated by KAE loaded NLCs. Co-administration of KAE-loaded nanoparticles and paclitaxel into cancer cells significantly strengthens the percentage of apoptosis $(p<0.05)$. Our results recommend that KAE incorporated into NLCs as an anti-cancer adjuvant is a powerful technique that may be a useful delivery system to enhance chemotherapy agents' effect on breast cancer cells.
\end{abstract}

Keywords: apoptosis; breast cancer; nanoparticle; Kaempferol.

(C) 2021 by the authors. This article is an open access article distributed under the terms and conditions of the Creative Commons Attribution (CC BY) license (https://creativecommons.org/licenses/by/4.0/).

\section{Introduction}

Kaempferol (KAE) (3,40,5,7-tetrahydroxyflavone) as flavonoid compounds exist in several natural products such as onions, red fruits [1]. Although Poor biodegradation and bioavailability are the two limitations of Kaempferol based on several scientific kinds of literature, it is a promising therapeutic flavonoid for cancer therapy, diabetes, and neurodegenerative diseases through oral administration [2, 3]. KAE has health benefits, including anti-cancer, anti-inflammatory, and antioxidant benefits associated with various signal transduction pathways [4]. According to previous studies, bioavailability and flavonoids' chemical stability are affected by water solubility and poor dissolution. Despite the progress in new pharmaceutical techniques, very few kaempferol delivery systems have been investigated to address kaempferol application limitations in cancer programs [5, 6]. Controlled release of 
flavonoids such as KAE loaded into nanocarriers can enhance the bioavailability and solubility of it and increase absorption and enhance stability against free radicals during food compounds' consumption and storage [7]. Efficient delivery of poorly water-soluble compounds and enhanced bioavailability are the two advantages of Lipidic nanoparticles. Solid lipid nanoparticles (SLNs) are responsible for the development of nanostructured lipid carriers (NLC) that are superior to SLNs due to their stability, improved drug release control, and higher drug loading capacity $[8,9]$. As such, to ameliorate the oral bioavailability of poorly soluble drugs, NLC draws scientific attention to a promising strategy [10]. The capability of NLC to improve oral absorption was demonstrated in studies of curcumin [11] and rapamycin [12]. However, the hindrance of its absorption in the intestine is affected by electrostatic repulsions between lipid bilayers the negatively charged surface of nanoparticles [13]. Recently, to achieve specific functions via augmenting oral bioavailability and intestinal absorption, complex surface-modified NLC has been designed [14]. Scientists widely investigated Chitosan (CS) as a natural cationic biopolymer to decorate the external surface of NLCs for drug delivery. Ability to open the tight connections in intestinal epithelial cells and adhere to mucosal surfaces are two factors that enhance drug absorption of CS [15]. In terms of drug delivery science, CS provides a great opportunity for the oral absorption of lipid nanoparticles, including SLN [16], NLC [17], and liposomes [18]. However, cellular and molecular properties of KAE-loaded lecithin/ chitosan nanoparticle (KAE-LC NP) systems have not yet been reported. Therefore, the present study aims to determine the formulation, physicochemical characterization, and in vitro anti-cancer mechanisms of KAE-LC NPs in MDA-MB468 breast cancer cells.

\section{Materials and Methods}

\subsection{Formulation of kaempferol-loaded NLCs.}

A modified hot homogenization method was harnessed to prepare KAE-loaded NLCs according to the following manufacture; a certain amount of KAE and Compritol and Miglyol were mixed and melted at 85 to become a homogeneous phase. To continue, the poloxamer as a surfactant was added in dropwise conditions due to the increased solubility of the solution. To continue, we used a sonicator to soak the sample for $3 \mathrm{~min}$. We allow the sample to became cool down at room temperature and got recrystallized. We applied chitosan oligosaccharides (COS) for targeting formulation. We liquified a certain amount of polymer in water to make a serial dilution of different concentrations of a water-soluble polymer, then we added with KAE dispersions.

\subsection{Optimization of kaempferol-loaded NLCs.}

The particle size and polydispersity index of KAE-NLCs were determined by the dynamic light scattering method. Malvern zeta analyzer was employed to measure the Zeta potential of Kaempferol-loaded NLCs. We used ultra-purified water to dilute samples to measure in triplicate condition. The visualization of nanoparticles' size and morphology was investigated by applying a scanning electron microscope SEM, Kyoto, Japan).

\subsection{Internalization of nanoparticles into the cell.}


We applied FITC as a dye label based on the lipids' weight $(5 \% \mathrm{w} / \mathrm{w})$. To separate the KAE-loaded NLCs from unloaded rhodamine B the Amicon ${ }^{\circledR}$ tube was used. To cultivate MDA-MB-468 cells $\left(4 \times 10^{4}\right.$ per well), we used six-well plates that previously were covered with $18 \mathrm{~mm}$ coverslips. Consequently, to observe the new formulation's penetration into cancer cells, we employed fluorescent microscopy (Olympus, Japan) during various times period (2080 minutes).

\subsection{Cell proliferation study.}

96-well microplates were used to cultivate MDA-MB-468 cells in a triplicate way at a different concentration by making serial dilution with 1, 2, 4, $8 \mathrm{nmol}$ paclitaxel and 10, 20, 40, 80 , and $160 \mu \mathrm{mol} / 1 \mathrm{KAE}$ for 24 and $48 \mathrm{~h}$. To substitute the medium in each well, we used $200 \mu \mathrm{l}$ fresh complete medium comprising $20 \mu \mathrm{l}$ MTT solution $(2 \mathrm{mg} / \mathrm{ml})$. Subsequently, the cells were incubated under the mentioned condition for about $3-4 \mathrm{~h}$ at $37^{\circ} \mathrm{C}$. Then, media/MTT mix was substituted with $200 \mu$ dimethyl sulfoxide $+25 \mu 1$ Sorenson's glycine buffer to each well. After 30 min shaking of the plate, we employed a 3200 microplate reader to measure absorbance at $570 \mathrm{~nm}[19]$.

\subsection{The evaluation of apoptosis by flow cytometry.}

Annexin V/FITC apoptosis detection kit was applied to whether the population of cancer cells goes to the early, late, and necrotic cell death or not. 6 well plate $\left(40 \times 10^{5}\right)$ was employed to seed MDA-MB-468 cells and treated with $1.75 \mathrm{nmol}$ paclitaxel, $45 \mu \mathrm{mol} \mathrm{KAE}$ and incubated at $37^{\circ} \mathrm{C}$, for 24 hours. We used trypsin to detach MDA-MB-468 cells from the plate, and then by using of PBS we washed suspended cells two times and next resuspended them with PBS. Finally, Annexin V/Propidium iodide (PI) was harnessed to stain cells for 15 min at room temperature in the dark. The determination of apoptotic cells was investigated by using a FACSCalibur flow cytometer.

\subsection{The investigation of apoptotic nuclei by DAPI.}

To detect the shape and morphology of MDA-MB-468 cells, DAPI staining was investigated. A 6-well plate $\left(300 \times 10^{3}\right)$ was applied to seed MDA-MB-468 cells. 3\% paraformaldehyde solution in PBS was used to fix the cells for $15 \mathrm{~min}$, and then $10 \mu \mathrm{g}$ DAPI in PBS was employed to stain cells at darkroom for another $20 \mathrm{~min}$ and then cells cleaned twice with PBS. In the end, fluorescent microscopy was used to assess the nuclear morphology of the cells.

\subsection{Reverse transcription-quantitative polymerase chain reaction ( $R T-q P C R)$.}

6-well plates were used to cultivate MDA-MB 468 cell lines and subjected to KAEloaded NLCs for $24 \mathrm{~h}$. The isolation of total RNA was done by using a TRIzol reagent. The nanoDrop device was employed to detect RNA quantification. To synthesis cDNA, one microgram of total RNA was carried out by Revert Aid Reverse Transcriptase (Thermo Fisher Scientific, Inc). The measurement of gene expression was done by Quantitative Real-time PCR methods by using specific primers (Table 1) and SYBR green PCR Master Mix Kit. To normalize the relative expression of each gene, a housekeeping gene was harnessed. 


\section{Results and Discussion}

\subsection{Characterization of kaempferol formulation into NLCs.}

Hot homogenization technique was harnessed to assemble KAE into NLCs systems. Particle size analyzer exhibited almost controlled size scattered of NLC nanoparticles in the range of 30-95 (Figure. 1A) nm that verified with SEM picture (Figure. 1C). The stability of nanoparticles with nearly +30 Zeta potential was confirmed and finite-size distribution with a polydispersity index (PDI) of 0.26 (Figure. 1B). Poloxamer is a surfactant that was injected in dropwise solution into KAE, and Compritol/Miglyol mixture led to the successful preparation of the KAE-NPs. Electrostatic interaction between the polycationic chitosan and negatively charged Compritol provided the stabile and spherical formation of nanoparticles. Chitosan enables to increase drug absorption, drug-solubility because of its hydrophilic molecular structure. Since nanoparticles' entrance into cancer cells' cell walls is through enhanced permeability and retention (EPR) mechanism, particle size plays an important role in determining anti-cancer activity. Thus, increased uptake of vehicles into cancer cells is highlighted by smaller particle size [20]. The other factor that plays an important role in nanoparticles' inhibitory effect is particle surface charge whereas, nanoparticles with the positively charged surface have improved interaction negatively charged surfaces of the cancer cell membrane [21]. The average size of nanoparticles obtained using KAE into formulation (30-100 nm) based on our experiment's conditions (Figure 1(A)). This size distribution of KAE gave rise to rescue formulation from aggregation and displayed high PDI (0.24). We observed that high concentrations of KAE arise to diminishes the distance between nanoparticles and lead to the blockage of the repulsive interaction between the nanoparticles and aggregation. The results were consistent with the previous studies [22,23]. The nanoparticle's surface charge affected nanoparticles' stability because of electrostatic interaction between particles determined by zeta potential (Figure 1B). The surface charge of the obtained colloidal particle ranges was $+30 \mathrm{mV}$ generated by the concentrations above of chitosan. These values are appropriate for a stable nanoparticle system because the range of $30 \mathrm{mV}$ is sufficient to prevent nanoparticle accumulation. We investigated the morphology of nanoparticles with Scanning electron micrographs. According to Figure $1 \mathrm{c}$, KAE-LC NPs in the optimal formulation have a spherical shape, uniformed, and polydisperse sizes from 30 to $150 \mathrm{~nm}$ with an average size of $75 \mathrm{~nm}$. Also, almost a little particle accumulation was observed during the drying process of the NPs.

\subsection{MTT assay experiments to assess the anti-proliferation effect of Kaempferol.}

Antiproliferative behavior of KAE, paclitaxel, and formulation was investigated under incubation with different concentrations of conditions above on MDA-MB468 breast cancer by the following 24 hours. As presented in Figure. 2 (A \& B), the $\mathrm{IC}_{50}$ values for $\mathrm{KAE}$ and paclitaxel were $44 \pm 0.52 \mu \mathrm{M}$ and $1.75 \pm 0.36 \mu \mathrm{M}$, respectively.

Kaempferol-loaded NLCs suppressed the proliferation of MDA-MB-468 cells more efficiently than Kaempferol alone ( $\mathrm{P}$ < 0.05). There is no conspicuous alteration between the breast cells treated with NLCs alone and untreated cells, which revealed that employed nanoparticles are safe and biocompatible with minimum toxicity (Figure 2c). The antiproliferation effect of Kaempferol against MDA-MB-468 cells was done to compare KAE alone and incorporation into nanoparticles. It is considered that the inhibitory dosage of KAE 
was $45 \mu \mathrm{M}$ comparable to the standard anti-neoplastic drug, paclitaxel, in particular, inhibition at $1.75 \mu \mathrm{M}$ of paclitaxel. According to our data, Kaempferol loaded nanoparticles enhanced the antitumor behavior of paclitaxel on the proliferation of MDA-MB-468 cells and a significantly lower dosage of paclitaxel needed against the inhibition of cancer cells.
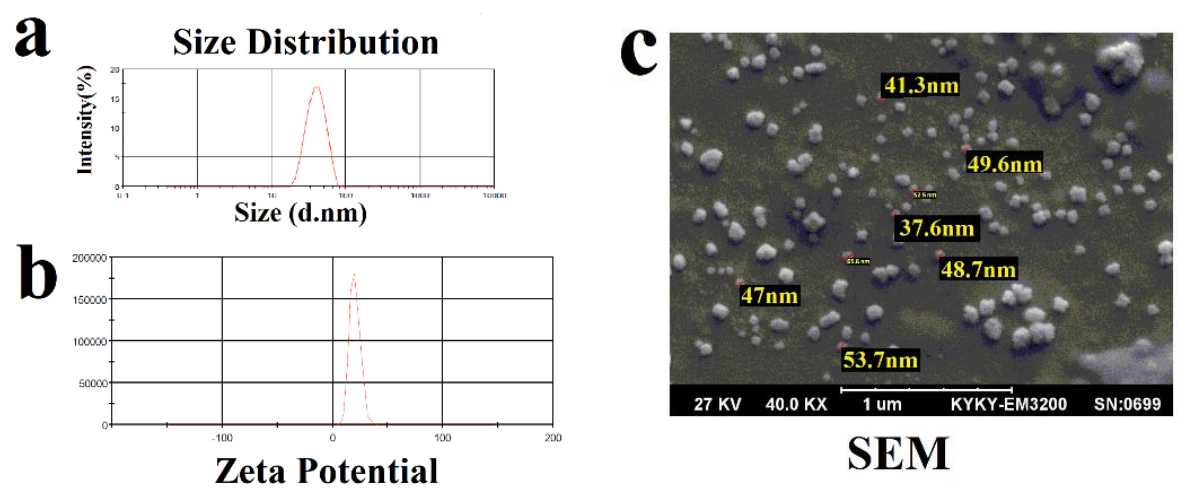

Figure 1. Nanoparticle Size scattering diagram (A) Distribution of Zeta potential histogram (B) Scanning electron microscopy (SEM) of the Kaempferol-loaded Nanostructured lipid carriers (NLCs) in aquatic solution (C).

Table 1. Primers for a reverse quantitative polymerase chain reaction.

\begin{tabular}{l|l}
\multirow{2}{*}{ Kene name } & Primer sequence (5'-3') \\
\hline \multirow{2}{*}{ BAD } & Forward: GAAAGAGTGGCAACCTGCCTTC \\
\cline { 2 - 2 } & Reverse: GCACCAAGTTTTACTACATCTGCC \\
\hline \multirow{2}{*}{ Bcl-2 } & Forward: GGAAGACGCTAGTGCTACAGA \\
\cline { 2 - 2 } & Feverse GAGCCTCCTTTGCCCAAGTTT \\
\cline { 2 - 2 } & Rerward: TACCGTCGTGACTTCGCAGAG \\
\hline \multirow{2}{*}{ MCl-1 } & Forward: AAC AAA GAG GCT GGG ATG \\
\cline { 2 - 2 } & Reverse: ATT GCA CTT ACA GTA AGG CTA TC \\
\hline \multirow{2}{*}{ s-actin } & Forward: TGCCCATCTACGAGGGGTATG \\
\cline { 2 - 2 } & Reverse: CTCCTTAATGTCACGCACGATTTC
\end{tabular}
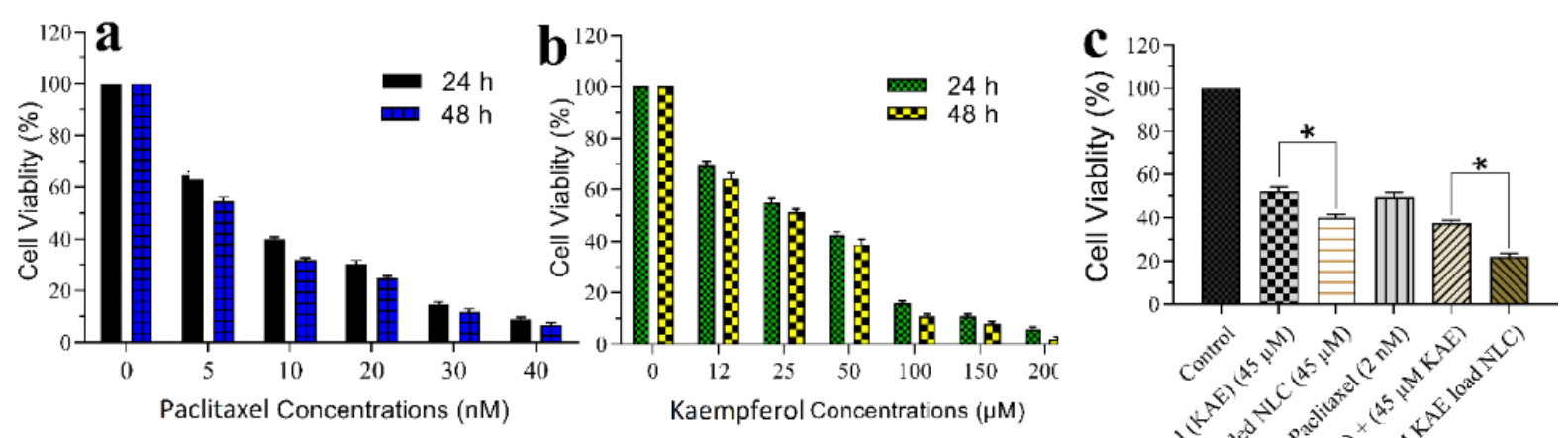

Figure 2. MDA-MB 468 mouse breast cancer cells were incubated with concentrations of 1-8nM Pac A), 10-160 $\mu \mathrm{M}$ Kaempferol B). Comparison among the rate of prevention of growth of MDA-MB468 mouse breast cancer cell lines with Paclitaxel, Kaempferol, and Kaempferol-loaded nanostructured lipid carriers (NLCs). Kaempferol plus Pac $44 \mu \mathrm{M}$ had more cytotoxicity than Kaempferol -NLC * $\mathrm{p}<0.05$ and Pac $1.75 \mathrm{nM}$ alone. Furthermore, Kaempferol-loaded NLCs plus Pac had significant cytotoxicity property than Kaempferol-loaded NLCs only**P<0.01. The outcome was shown as the mean $\pm \mathrm{SD}(\mathrm{n}=3)$. 
There is no substantial alteration in the cancer cell populations when treated with NLCs alone and control(untreated) cells (Figure 3). Incubation MDA-MB468 breast cells with paclitaxel alone increased the percentage of apoptotic cells by $10.4 \%$. At the same time, treatment with Kaempferol -loaded NLCs upsurge the percentage of apoptotic phase to $16.2 \%$. In combination with paclitaxel, this percent reached up to $22 \%$., The cell cycle arrest experiment confirmed the apoptosis results, whereas incubation MDA-MB468 cells with KAE loaded NLCs stopped cells in Sub G1 phase up to $19 \%$ while KAE exhibited only $15 \%$ cell cycle arrest (Figure 4). KAE, paclitaxel, and Kaempferol loaded-NLCs had significant antitumor behavior against tumor cell structure, such as perturbation in a spherical shape and transforming cancer cells shrinkage form, which has approved disintegration of nuclei along with initiation program cell death. In a study, Kaempferol showed a concentration-dependent antiproliferative effect on SiHa cells [24]. In another study, Kaempferol exhibited a significant antitumor effect by stopping the cell cycle and inducing apoptosis in HeLa cells [25]. In the same study, the inhibitory effect of kaempferol growth showed that the PI3K/Akt pathway unregulated in various different cancers [26]. Other studies have shown that Kaempferol inhibits prostate [27] and lung [28] cancer cells by upregulation of caspases (3, 8, and 9) and bladder cancer cells based on a PTEN activation mechanism [29]. In one study, apoptotic death through activating PARP cleavages in renal cancer cells was induced by kaempferol [30]. So, we investigated the programmed cell death mechanism(apoptosis) of KAE loaded nanoparticles in MDA-MB 468 to evaluate whether formulation can elevate the efficacy of KAE or not. As shown in Figure 3, nanoparticles alone had no cytotoxic effect against cancer cells, indicating that these drug carriers are safe and biocompatible. Furthermore, treatment cancer cells with KAE loaded nanoparticles increased early apoptosis from 0.1 up to 6 percent compared to KAE alone. KAE loaded nanoparticles and paclitaxel showed the highest percentage of apoptosis compared with KAE combined with paclitaxel which indicates the synergistic effect of nanoparticles in delivering drugs to cancer cells. KAE loaded nanoparticles revealed $11 \%$ apoptosis that was the highest from both KAE and paclitaxel. Because different mechanisms are involved in the phenomenon of apoptosis in different tumor cells, the identification signal pathway of Kaempferol that causes cell death requires further investigation. KAE is endowed with great potential in cancer prevention due to the bioavailability, low-cost, and safety properties. To understand the cell cycle arrest mechanism of this formulation, we did a flow cytometric assay and noticed that KAE-loaded nanoparticles arrested the population of cancer cells up to $21 \%$ in the Sub G1 phase, while 23.15 percent of cells accumulated in the $\mathrm{G} 2 / \mathrm{M}$ phase.

\subsection{Nanoparticle internalization study and DAPI staining by fluorescent microscope.}

The interaction between the lipidic structure of formulation and cancer cell membrane made it possible to investigate quantitative KAE loaded nanoparticles internalization into breast cells to endocytosis phenomenon. The accumulation of KAE into cancer cells was measured based on intensification of rhodamine B as track dye in MDA-MB-468 cells from 20 to $80 \mathrm{~min}$ interval times (Figure. $5 \mathrm{~A}$ ). The identification of the apoptotic body and confirmation flow cytometric analysis in the quantification experiments in figure 3, DAPI staining, was done. As shown in (Figure. 5 B) 


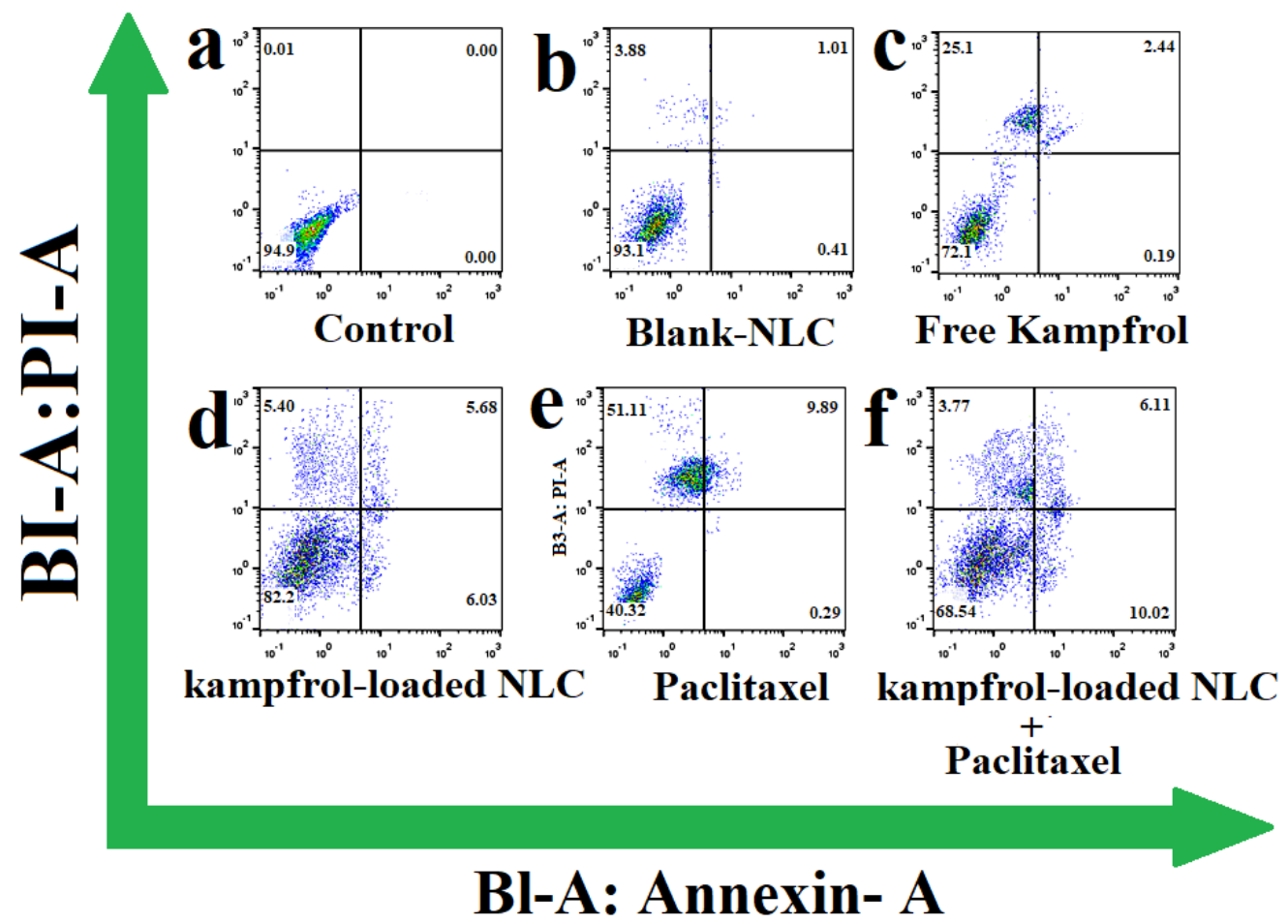

Figure 3. Kaempferol-loaded Nanostructured lipid carriers (NLCs) increase the early phase of apoptosis in MDA-MB 468 cancer cells. Cells were treated with $\mathrm{IC}_{50}$ of Kaempferol, paclitaxel and Kaempferol -loaded NLCs. mean \pm standard deviation $(n=3)$ was applied for presenting data. (A) Control group; (B) Kaempferol; (C) Nano blank; (D) paclitaxel; (E) Kaempferol -loaded NLCs; (F) Kaempferol -loaded NLCs and paclitaxel.

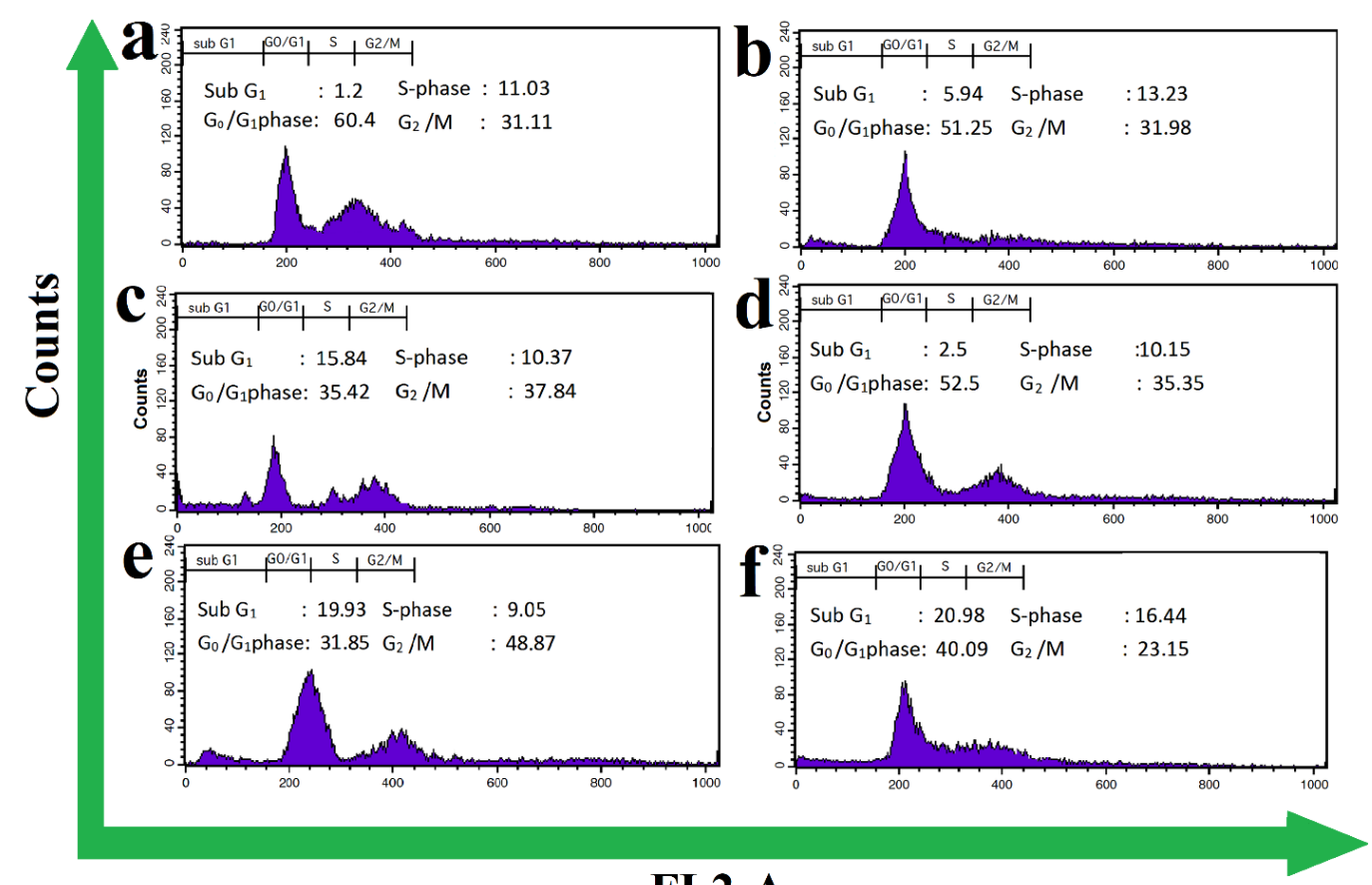

FL2-A

Figure 4. Effects of KAE-loaded NLC on cell cycle distribution of MDA-MB-468 cells. a) Control group, b) Nano blank; c) KAE; d) Paclitaxel (Pac); e) Formulation; f) Formulation+ Pac. 

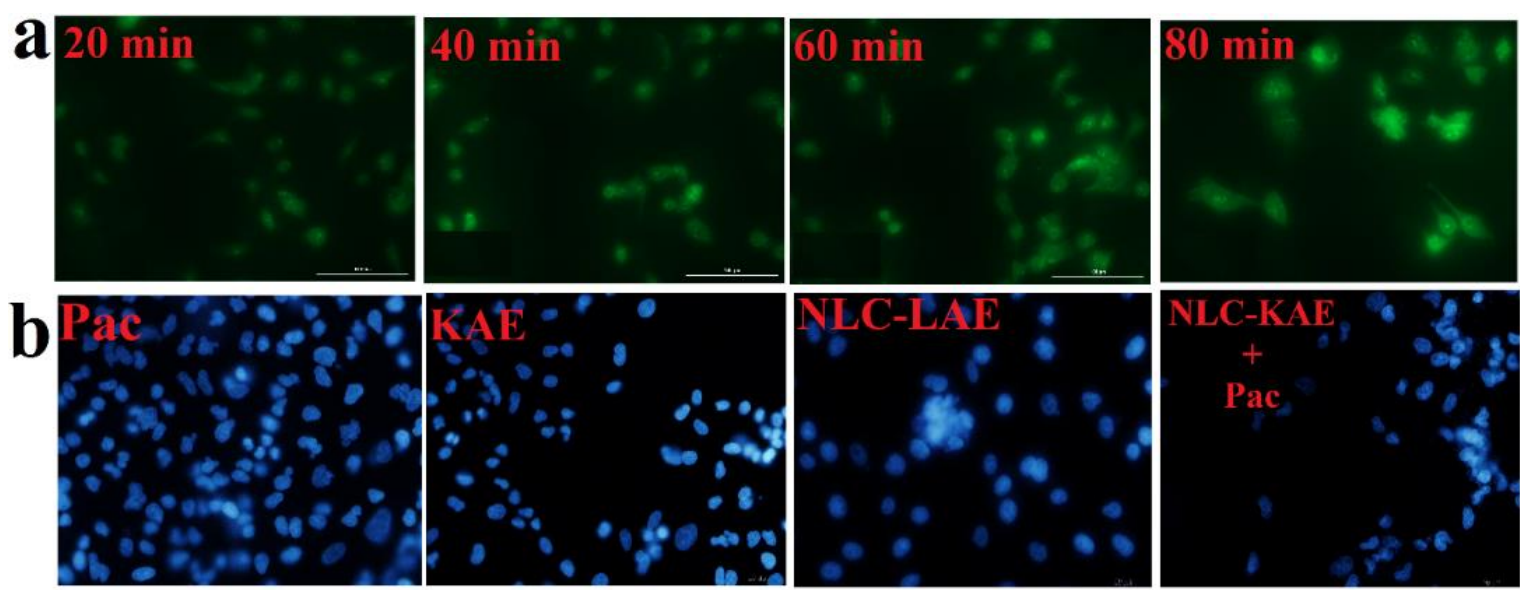

Figure 5. Cellular internalization was evaluated by rhodamine B dye mixed during formulation preparation and monitoring cell uptake procedure between 20 up to 80 minutes' time intervals confirmed penetrability and retention of formulations comprising Kaempferol in MDA-MB468 mouse breast cancer cell (A). Fluorescence microscopy was applied to illustrate the morphological alteration of MDA-MB468 mouse breast cancer cells under incubation with DAPI staining (B).

\subsection{Real-time PCR.}

Anti-apoptotic and proapoptotic pathway genes were investigated to clarify more extensive validation that kaempferol-NLCs have engaged in apoptosis pathway in breast MDAMB468 cells. kaempferol-NLCs, compared with KAE alone incubation, dramatically narrowed the expression level of KI-67, MCl-1, and bcl-2. On the other hand, in comparison to the control group expression of a proapoptotic gene, BAD mRNA was also meaningfully increased after treatment with KAE-NLCs $(\mathrm{P}<0.05)$ (Figure 6). By the way, nano blank had no alteration in the level of expression, whether pro or anti-apoptotic genes. To verify that which genes are involved in initiating the apoptotic pathway under treatment with KAE loaded nanoparticles, Real-time PCR was investigated. Our results showed that KAE-loaded nanoparticles decreased mcl-1 and bcl 2 gene expression and increased BAD as proapoptotic genes. We checked the level of ki-67 as proliferation index in cells under incubation with different conditions and noticed that our formulation was able to diminish the expression of ki-67 levels significantly in comparison with other groups.

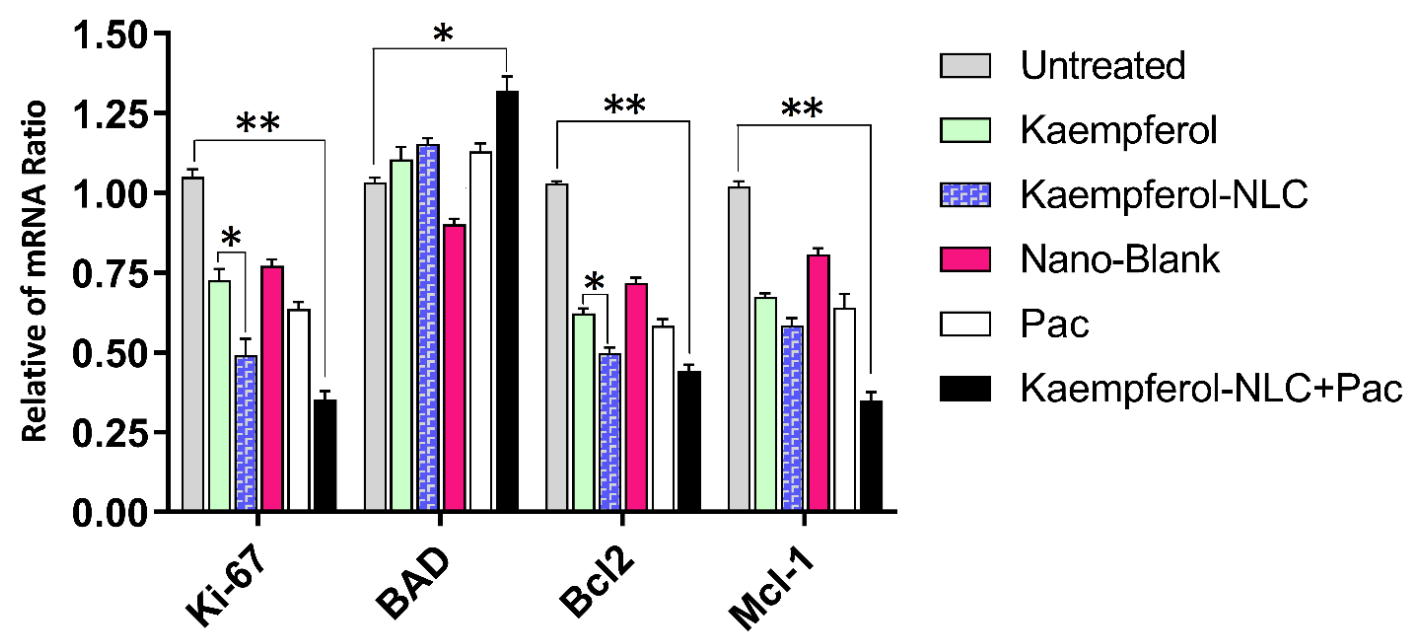

Figure 6. MDA-MB-468 breast cells cultivated with a designed concentration of KAE and KAE-loaded NLCs for $24 \mathrm{~h}$. The expression pattern of KI-67, MCl-1, bcl-2 and Bad genes in groups of study determined using RealTime PCR. Mean \pm standard deviation $(\mathrm{n}=3)$ was shown for presenting data. ${ }^{*} \mathrm{p}<0.05, * * \mathrm{p}<0.01$, s compared with untreated as control value. 
Therefore, a natural, non-toxic agent can be useful when being loaded into effective drug delivery systems against cancer cells, whether alone or in combination with first-line chemotherapeutic agents.

\section{Conclusions}

Kaempferol-loaded NLCs amplified the cytotoxicity of paclitaxel against MDA-MB 468 mouse breast cancer cells. Our data also highlighted that paclitaxel could be enriched in the synergistic antitumor behavior when combined with kaempferol-loaded NLCs by inhibiting apoptotic signaling and suppression of cancer cell cycle arrest in Sub G1 arrest as well as downregulated its anti-apoptotic Bcl-2 family genes levels. By considering all results, paclitaxel co-treatment with kaempferol-loaded NLCs as adjuvant can produce a more efficient breast cancer treatment.

\section{Funding}

This work was supported by grants from "Molecular Medicine Research Center, Tabriz University of Medical Science".

\section{Acknowledgments}

We would like to thanks Dr. Bahman Ramezani for providing some useful software infrastructure to accomplish this work.

\section{Conflicts of Interest}

The authors declare no conflict of interest.

\section{References}

1. Namdeo, A.G.; Brgeoddu, S.H.; Amawi, H.; Ashby, Jr.C.R.; Tukaramrao, D.B.; Trivedi, P.; Babu, R.J.; Tiwari, A.K. Flavonoids as Multi-Tat Compounds: A Special Emphasis on their Potential as Chemoadjuvants in Cancer Therapy. Current pharmaceutical design 2020, 26, 1712-1728, https://doi.org/10.2174/1381612826666200128095248.

2. Pang, X.; Zhang, X.; Jiang, Y.; Su, Q.; Li, Q.; Li, Z. Autophagy: Mechanisms and Therapeutic Potential of Flavonoids in Cancer. Biomolecules 2021, 11, https://doi.org/10.3390/biom11020135.

3. Lokhande, K.B.; Ballav, S.; Thosar, N.; Swamy, K.V.; Basu, S. Exploring conformational changes of PPAR$\mathrm{V}$ complexed with novel Kaempferol, quercetin, and resveratrol derivatives to understand binding mode assessment: a small-molecule checkmate to cancer therapy. Journal of Molecular Modeling 2020, 26, 1-12, https://doi.org/10.1007/s00894-020-04488-0.

4. Ashrafizadeh, M.; Tavakol, S.; Ahmadi, Z.; Roomiani, S.; Mohammadinejad, R.; Samarghandian, S. Therapeutic effects of Kaempferol affecting autophagy and endoplasmic reticulum stress. Phytotherapy Research 2020, 34, 911-923, https://doi.org/10.1002/ptr.6577.

5. Ilk, S.; Saglam, N.; Özgen, M. Kaempferol loaded lecithin/chitosan nanoparticles: Preparation, characterization, and their potential applications as a sustainable antifungal agent. Artificial cells, nanomedicine, and biotechnology 2017, 45, 907-916, https://doi.org/10.1080/21691401.2016.1192040.

6. Kahroba, H.; Ramezani, B.; Maadi, H.; Sadeghi, M.R.; Jaberie, H.; Ramezani, F. The role of Nrf2 in Neural stem/Progenitors cells: from maintaining stemness and self-renewal to promoting differentiation capability and facilitating therapeutic application in neurodegenerative disease. Ageing Research Reviews 2020, 65, https://doi.org/10.1016/j.arr.2020.101211.

7. More, M.P.; Pardeshi, S.R.; Pardeshi, C.; Sonawane, G.A.; Shinde, M.N.; Deshmukh, P.K.; Naik, J.B.; Kulkarni, A.D. Recent advances in phytochemical based Nano-formulation for drug resistant Cancer. Medicine in Drug Discovery 2021, 10, https://doi.org/10.1016/j.medidd.2021.100082.

8. Raeisi, S.; Chavoshi, H.; Mohammadi, M.; Ghorbani, M.; Sabzichi, M.; Ramezani, F. Naringenin-loaded nanostructured lipid carrier fortifies oxaliplatin-dependent apoptosis in HT-29 cell line. Process Biochemistry 2019, 83, 168-175, https://doi.org/10.1016/j.procbio.2019.05.013. 
9. Khaki-Khatibi, F.; Zeinali, M.; Ramezani, B.; Sabzichi, M.; Mohammadian, J.; Hamishehkar, H. Harnessing WYE-132 as an inhibitor of the mTOR signaling enriches the cytotoxicity effect of vinblastine in B16F10 melanoma cancer cells. Process Biochemistry 2020, 99, 123-130, https://doi.org/10.1016/j.procbio.2020.08.026.

10. Salehi, B.; Cruz-Martins, N.; Butnariu, M.; Sarac, I.; Bagiu, I.-C.; Ezzat, S.M.; Wang, J.; Koay, A.; Sheridan, H.; Adetunji, C.O. Hesperetin's health potential: moving from preclinical to clinical evidence and bioavailability issues, to upcoming strategies to overcome current limitations. Critical Reviews in Food Science and Nutrition 2021, 1-16, https://doi.org/10.1080/10408398.2021.1875979.

11. Rapalli, V.K.; Kaul, V.; Waghule, T.; Gorantla, S.; Sharma, S.; Roy, A.; Dubey, S.K.; Singhvi, G. Curcumin loaded nanostructured lipid carriers for enhanced skin retained topical delivery: optimization, scale-up, invitro characterization and assessment of ex-vivo skin deposition. European Journal of Pharmaceutical Sciences 2020, 152, https://doi.org/10.1016/j.ejps.2020.105438.

12. Landh, E.; Moir, L.M.; Dos Reis, L.G.; Traini, D.; Young, P.M.; Ong, H.X. Inhaled rapamycin solid lipid nano particles for the treatment of Lymphangioleiomyomatosis. European Journal of Pharmaceutical Sciences 2020, 142, https://doi.org/10.1016/j.ejps.2019.105098.

13. Fathi, M.; Vinceković, M.; Jurić, S.; Viskić, M.; Režek Jambrak, A.; Donsì, F. Food-grade colloidal systems for the delivery of essential oils. Food Reviews International 2021, 37, 1-45, https://doi.org/10.1080/87559129.2019.1687514.

14. Mahmoudi, S.; Ghorbani, M.; Sabzichi, M.; Ramezani, F.; Hamishehkar, H.; Samadi, N. Targeted hyaluronic acid-based lipid nanoparticle for apigenin delivery to induce Nrf2-dependent apoptosis in lung cancer cells. Journal of Drug Delivery Science and Technology 2019, 49, 268-276, https://doi.org/10.1016/j.jddst.2018.11.013.

15. Wang, J.; Kong, M.; Zhou, Z.; Yan, D.; Yu, X.; Cheng, X.; Feng, C.; Liu, Y.; Chen, X. Mechanism of surface charge triggered intestinal epithelial tight junction opening upon chitosan nanoparticles for insulin oral delivery. Carbohydrate polymers 2017, 157, 596-602, https://doi.org/10.1016/j.carbpol.2016.10.021.

16. Maretti, E.; Pavan, B.; Rustichelli, C.; Montanari, M.; Dalpiaz, A.; Iannuccelli, V.; Leo, E. Chitosan/heparin polyelectrolyte complexes as ion-paring approach to encapsulate heparin in orally administrable SLN: In vitro evaluation. Colloids and Surfaces A: Physicochemical and Engineering Aspects 2021, 608, https://doi.org/10.1016/j.colsurfa.2020.125606.

17. Ling, T.J.S.; Roberts, C.J.; Billa, N. Mucoadhesive chitosan-coated nanostructured lipid carriers for oral delivery of amphotericin B. Pharmaceutical development and technology 2019, 24, 504-512, https://doi.org/10.1080/10837450.2018.1515225.

18. Miao, Y.-Q.; Chen, M.-S.; Zhou, X.; Guo, L.-M.; Zhu, J.-J.; Wang, R.; Zhang, X.-X.; Gan, Y. Chitosan oligosaccharide modified liposomes enhance lung cancer delivery of paclitaxel. Acta Pharmacologica Sinica 2021, 1-9, https://doi.org/10.1038/s41401-020-00594-0.

19. Ghorbani, M.; Sabzichi, M.; Ramezani, F.; Mohammadian, J. Adjuvant therapy with stattic enriches the antiproliferative effect of doxorubicin in human ZR-75-1 breast cancer cells via arresting cell cycle and inducing apoptosis. Biomedicine \& Pharmacotherapy 2019, 109, 1240-1248, https://doi.org/10.1016/j.biopha.2018.10.183.

20. Rejinold, N.S.; Han, Y.; Yoo, J.; Seok, H.Y.; Park, J.H.; Kim, Y.-C. Evaluation of cell penetrating peptide coated $\mathrm{Mn}$ : ZnS nanoparticles for paclitaxel delivery to cancer cells. Scientific reports 2018, 8, 1-11, https://doi.org/10.1038/s41598-018-20255-x.

21. Salama, A.; Hasanin, M.; Hesemann, P. Synthesis and antimicrobial properties of new chitosan derivatives containing guanidinium groups. Carbohydrate Polymers 2020, https://doi.org/10.1016/j.carbpol.2020.116363.

22. Barbieri, S.; Sonvico, F.; Como, C.; Colombo, G.; Zani, F.; Buttini, F.; Bettini, R.; Rossi, A.; Colombo, P. Lecithin/chitosan controlled release nanopreparations of tamoxifen citrate: loading, enzyme-trigger release and cell uptake. Journal of Controlled Release 2013, 167, 276-283, https://doi.org/10.1016/j.jconrel.2013.02.009.

23. Souza, M.P.; Vaz, A.F.; Correia, M.T.; Cerqueira, M.A.; Vicente, A.A.; Carneiro-da-Cunha, M.G. Quercetin-loaded lecithin/chitosan nanoparticles for functional food applications. Food and bioprocess technology 2014, 7, 1149-1159, https://doi.org/10.1007/s11947-013-1160-2.

24. Tu, L.Y.; Bai, H.H.; Cai, J.Y.; Deng, S.P. The mechanism of Kaempferol induced apoptosis and inhibited proliferation in human cervical cancer SiHa cell: From macro to nano. Scanning 2016, 38, 644-653, https://doi.org/10.1002/sca.21312.

25. Alonso-Castro, A.J.; Ortiz-Sánchez, E.; García-Regalado, A.; Ruiz, G.; Núñez-Martínez, J.M.; GonzálezSánchez, I.; Quintanar-Jurado, V.; Morales-Sánchez, E.; Dominguez, F.; López-Toledo, G. Kaempferitrin induces apoptosis via intrinsic pathway in HeLa cells and exerts antitumor effects. Journal of ethnopharmacology 2013, 145, 476-489, https://doi.org/10.1016/j.jep.2012.11.016.

26. Mirza-Aghazadeh-Attari, M.; Ekrami, E.M.; Aghdas, S.A.M.; Mihanfar, A.; Hallaj, S.; Yousefi, B.; Safa, A.; Majidinia, M. Targeting PI3K/Akt/mTOR signaling pathway by polyphenols: Implication for cancer therapy. Life sciences 2020, 255, https://doi.org/10.1016/j.lfs.2020.117481. 
27. Halimah, E.; Diantini, A.; Destiani, D.P.; Pradipta, I.S.; Sastramihardja, H.S.; Lestari, K.; Subarnas, A.; Abdulah, R.; Koyama, H. Induction of caspase cascade pathway by kaempferol-3-O-rhamnoside in LNCaP prostate cancer cell lines. Biomedical reports 2015, 3, 115-117, https://doi.org/10.3892/br.2014.385.

28. Kumar, A.N.; Bevara, G.B.; Kaja, L.K.; Badana, A.K.; Malla, R.R. Protective effect of 3-O-methyl quercetin and Kaempferol from Semecarpus anacardium against $\mathrm{H} 2 \mathrm{O} 2$ induced cytotoxicity in lung and liver cells. BMC complementary and alternative medicine 2016, 16, 1-13, https://doi.org/10.1186/s12906-016-1354-z.

29. Ashrafizadeh, M.; Zarrabi, A.; Samarghandian, S.; Najafi, M. PTEN: What we know of the function and regulation of this onco-suppressor factor in bladder cancer? European Journal of Pharmacology 2020, 881, https://doi.org/10.1016/j.ejphar.2020.173226.

30. Alugoju, P.; Chaitanya, N.S.; Swamy, V.K.; Kancharla, P.K. Phytotherapy for breast cancer. In: A Theranostic and Precision Medicine Approach for Female-Specific Cancers. Elsevier: 2021; pp. 129163, https://doi.org/10.1016/B978-0-12-822009-2.00007-8. 